\title{
Toxicidad aguda de una formulación comercial de glifosato sobre Poecilla reticulata (pisces: poecilidae) en condiciones de laboratorio
}

\author{
Acute toxicity of a comercial glyphosate \\ formulation on Poecilla reticulata (pisces: \\ poecilidae) in laboratory conditions
}

\author{
Francois Herrera Jacquelin ${ }^{\star}$, Karenn Eliana Beltran González ${ }^{\star \star}$, Jennifer \\ Andrea Garzón González, Laura González Sánchez ${ }^{\star \star \star}$, Laura Milena Herrera \\ Rivera $^{\dagger}$, Andrés Felipe Torres Cequerra ${ }^{\ddagger}$ \\ Universidad Santo Tomás
}

FECHA DE ENTREGA: 18 DE FEBRERo DE 2016

FECHA DE EVAluación: 8 DE ABRIL DE 2016

FECHA DE APROBACIÓN: 2 DE MAYO DE 2016

\begin{abstract}
Resumen El glifosato es un herbicida ampliamente usado en Colombia tanto en actividades agrícolas como para la erradicación de cultivos ilícitos. La deriva por viento en aspersiones aéreas y la lixiviación de este producto pueden contaminar los cuerpos de agua por lo que es importante medir su efecto tóxico. Poblaciones del pez Poecilla reticulata fueron sometidas a concentraciones de $0 ; 2.3 ; 5.5 ; 8.3 ; 11.0 ; 13.8 \mathrm{mg}$ de glifosato/L en la formulación comercial Roundup ${ }^{\circledR} 747$ SG /L, para determinar la mortalidad de los individuos tratados y la concentración letal $50\left(\mathrm{CL}_{50}\right)$ en condiciones de laboratorio. La mortalidad fue evaluada a las 24, 48, 72 y 96 horas encontrándose que esta aumentó con la concentración del herbicida y con el tiempo transcurrido. También se observaron alteraciones en el patrón de nado de los peces estudiados El valor de la concentración letal 50 del glifosato sobre $P$. reticulata a las 96 horas de aplicados los tratamientos fue de $3.14 \mathrm{mg} / \mathrm{L}$, lo que sugiere un impacto ecológico en ecosistemas naturales y un uso potencial de esta especie de pez como biondicador de contaminación para este herbicida en ensayos de toxicidad aguda.
\end{abstract}

Abstract Glyphosate is an herbicide that is widely used in Colombia not only for farming, but also for illicit crop eradication. Wind drift in air spraying and the lixiviation of this product can contaminate water bodies

\footnotetext{
* francoisherrera@usantotomas.edu.co

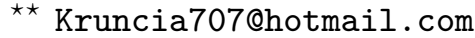

$\star \star \star ~ l a u r a g o n z a l e z @ u s a n t o t o m a s . e d u . c o$

$\dagger$ laumi1146@yahoo.com

$\ddagger$ andrestorresc@usantotomas.edu.co
} 
and for this reason, it is important to measure its toxic effect. Fish such as the Poecilla reticulata were submitted to concentrations of $0 ; 2.3 ; 5.5$; $8.3 ; 11.0 ; 13.8 \mathrm{mg}$ of glyphosate/L in the commercial formula Roundup ${ }^{\circledR}$ $747 \mathrm{SG} / \mathrm{L}$ to determine the mortality of treated individuals and the lethal concentration $50\left(\mathrm{LC}_{50-96}\right)$ in lab conditions. Mortality was tested at 24, 48,72 , and 96 hours, and we were able to find that it increased as the herbicide concentration and the time elapsed increased. We also noticed a variation in the swimming pattern of the fish studied. The value of the 50-lethal concentration glyphosate over P. reticulata during the first 96 hours that the treatment was applied was of $3.14 \mathrm{mg} / \mathrm{L}$, which suggests an ecological impact in natural ecosystems and a potential use of this type of fish as a contamination bioindicator for this herbicide in extreme toxicity tests.

Palabras Clave: Bioensayo, concentración letal 50, glifosato, Poecilla reticulata, toxicidad aguda, roundup

Keywords: Bioassay, lethal concentration 50, glyphosate, Poecilla reticulate, acute toxicity, roundup

\section{Introducción}

El glifosato (N-fosfonometilglicina, $\mathrm{C}_{3} \mathrm{H}_{8} \mathrm{NO}_{5} \mathrm{P}$ ) es un herbicida sistémico no selectivo desarrollado en los años 70 y originalmente comercializado bajo la marca Roundup® de la empresa Monsanto [1] [2], [3]. Actualmente, se distribuyen diferentes presentaciones comerciales como Rodeo®, Accord ${ }^{\circledR}$, Kleen up ${ }^{\circledR}$, entre otros [4]. Este herbicida de muy baja presión de vapor y alta solubilidad en agua actúa inhibiendo la 5-enolpiruvil-shiquimato-3-fosfato sintetasa (EPSPS), enzima responsable de la formación de los aminoácidos aromáticos fenilalanina, tirosina y triptófano [1].El glifosato es un producto ampliamente usado a nivel mundial, se estima que se consumen unas 700,000 toneladas de este herbicida al año [5]. En Colombia se utiliza principalmente en actividades agrícolas: como secante de granos, madurante de la caña de azúcar y como herbicida en cultivos de arroz, pasto entre otros [4], también se utiliza en aspersiones aéreas para la erradicación de cultivos de coca y amapola desde el año 1992. Se reporta una aplicación acumulada en estos programas de 1,420,130 litros hasta el año 2004 y un área asperjada de 104.771 ha para el año 2009 [6] [7]. Como consecuencia de la deriva (movilización del herbicida por viento a áreas no blanco) y de la lixiviación, el glifosato puede contaminar las fuentes de agua [8]. Se ha evaluado su toxicidad en varias especies acuáticas indicadoras como bacterias, algas microscópicas, micro-crustáceos, anfibios y peces. La literatura reporta concentraciones letales 50 de glifosato en peces en un rango muy amplio que va de10 a $210 \mathrm{mg} / \mathrm{L}$ para glifosato puro (grado técnico) y de 10 a $1000 \mathrm{mg} / \mathrm{L}$ en caso de glifosato en fórmulas comerciales (para doce especies de peces estudiadas y reportadas en múltiples fuentes) [9] [10]. Estudios realizados en Colombia reportan $\mathrm{CL}_{50-96}$ de $97.47 \mathrm{mg} / \mathrm{L}$ de glifosato para juveniles de cachama [8] [11] además de efectos en el sistema inmune y sensorial al ser expuestos a dosis subletales [12] [13]. La 
concentración letal reportada para microcrustaceos (Daphnia spp y Gammarus sp) es de 5.3 a $930 \mathrm{mg} / \mathrm{L}$ (para glifosato formulado) y de 44 a $5600 \mathrm{mg} / \mathrm{L}$ en ninfas de chiromonidos (Insecta, Diptera) [3] [2]. Folmar (1979) reporta una contribución importante del surfactante POEA, con concentraciones letales de dos a tres órdenes de magnitud inferiores al del glifosato puro pero similar al del Roundup ${ }^{\circledR}$ en trucha arcoíris y en pez luna de agallas azules, principalmente en aguas duras [9] [4].

Poecilla reticulata (Familia Poecilidae), es un pez de agua dulce conocido como gupy, cuya distribución natural abarca las Antillas, Mesoamérica y el norte de América del Sur: Venezuela, el norte de Brasil, Guyana Británica y Suriname. Esta especie es ampliamente cultivada y utilizada por acuaristas del mundo como pez ornamental o como alimento vivo para especies de peces más grandes por lo que actualmente su distribución es cosmopolita [14] [15]. Su manejo como indicador biológico presenta diversas ventajas: es fácil de obtener (su crianza en rápida y barata o se puede conseguir en criaderos comerciales de peces) es fácil de transportar y es muy resistente a condiciones de altas densidades poblacionales y bajas concentraciones de oxígeno. El presente estudio pretende evaluar la concentración letal $50\left(\mathrm{CL}_{50}\right)$ del glifosato en su presentación comercial de Roundup ${ }^{\circledR} 747$ SG en el gupy bajo condiciones de laboratorio, en búsqueda de modelos biológicos como bioindicadores de toxicidad por este tipo de compuestos.

\section{Metodología}

\subsection{Sujetos experimentales}

Para el experimento siguiendo los lineamientos para ensayos de toxicidad aguda de la OCDE [16] se utilizaron individuos jóvenes de cuatro a seis semanas de edad provenientes de una cría comercial privada y de diferentes desoves. Los individuos adquiridos, alrededor de 300, fueron trasladados al laboratorio de la Universidad Santo Tomás de Bogotá y mantenidos en dos acuarios de vidrio de 84 litros. Cada acuario tenía un aireadores, un filtro, luz natural, a una temperatura de $20 \pm 1$ grados Celsius, y se alimentaron con una dieta comercial en forma de escamas, tres escamas diarias por pecera. Los peces se mantuvieron bajo estas condiciones durante 6 días antes del montaje del ensayo. Se hizo una selección de los individuos $(n=180)$ de 2 a $2.5 \mathrm{~cm}$ de longitud ya que en ensayos preliminares se observó que estos fueron los que resistían mejor las condiciones experimentales.

\subsection{Condiciones experimentales}

Después del periodo de aclimatación antes descrito los peces fueron trasladados a los recipientes que contenían el agua con diferentes concentraciones de glifosato. Para el ensayo definitivo se utilizó como unidad experimental recipientes de PET (politereftalato de etileno) transparente con capacidad de $2 \mathrm{~L}$, con 10 individuos cada uno. Durante el tiempo del ensayo no se usaron aireadores en los recipientes ni se alimentaron los peces y el agua se mantuvo a una temperatura de $20 \pm 1$ 
${ }^{\circ} \mathrm{C}$.. El agua utilizada fue de la llave madurada durante 72 horas con un $\mathrm{pH}$ de 7.4 y una dureza total de $53 \mathrm{mg} / \mathrm{L}$. Estas condiciones experimentales se basaron en ensayos preliminares en donde se evaluaron diferentes densidades de peces en un volumen de dos litros, encontrando que 10 individuos podían sobrevivir durante más de cinco días sin alimentación ni aireación. Toda manipulación se hizo utilizando una red de acuario de $5 \times 7 \mathrm{~cm}$ tratando de minimizar el estrés de los individuos.

\subsection{Determinación de la $\mathrm{CL}_{50}$}

Para los ensayos se utilizó como fuente del glifosato el producto Roundup ${ }^{\circledR} 747$ SG; sal de monoamonio de N fosfonometilglicina a una concentración de $74.7 \%$ peso/peso. En ensayos preliminares se determinó que una concentración de 15 mg de Roundup® 747 SG /L causaban la muerte de la mayoría de individuos en un periodo menor a 24 horas. Basado en este hallazgo se establecieron seis tratamientos: un control sin glifosato y cinco concentraciones del producto comercial $(3,1 ; 7,4 ; 11,0 ; 14,7$ y 18,5 mg Roundup® 747 SG/L. La concentración del ingrediente activo para estos tratamientos fue de $2.3 ; 5.5 ; 8.2 ; 11.0 ; 13.8 \mathrm{mg}$ glifosato/L respectivamente. Para cada tratamiento se montaron tres recipientes con diez individuos cada uno para un total de 30 individuos/tratamiento. Los tratamientos se observaron inmediatamente después de aplicados durante una hora y posteriormente cada 12 horas. La mortalidad de los peces fue registrada a las 24, 48, 72 y 96 horas después de aplicados los tratamientos y se hizo una descripción cualitativa de las alteraciones de nado de los sujetos experimentales.

\subsection{Análisis estadístico}

Los datos fueron analizados mediante la prueba no paramétrica Kruskall-Wallis, posterior a la validación de los supuestos estadísticos de normalidad (i.e. prueba de Kolmogorov-Smirnov). para determinar las diferencias para la mortalidad de los individuos entre los tratamientos a las 96 horas de su aplicación. La $\mathrm{CL}_{50-96}$ se usó regresión logística (modelo logit) usando el programa estadístico pro-stat.

\section{Resultados y discusión}

Durante las primeras horas de la aplicación de los tratamientos los peces expuestos a 11 y $13.8 \mathrm{mg} / \mathrm{L}$ de glifosato, mostraron signos de híperexcitación caracterizados por un nado a una velocidad mayor a la normal en el tercio superior del recipiente, similar a lo observado en los ensayos preliminares y consistente con los daños neurotóxicos citados en la literatura, [17], [18] [19], no obstante, dichos daños no fueron letales inmediatos. Posterior a las 24 horas se presentó una disminución de la actividad de nado, caracterizada por movimientos lentos y un patrón de nado alterado (nado lateral). En los tratamientos con concentraciones más bajas $(2.3 ; 5.5 ; 8.2 \mathrm{mg} / \mathrm{L})$ no se observaron cambios inmediatos en el patrón de nado, sin embargo en las observaciones a partir de las 24 horas se evidenció el letargo 
de los individuos. No se observaron alteraciones en el nado en las unidades experimentales correspondientes al testigo.

En todos los tratamientos se observó una mortalidad dependiente del tiempo. En el grupo control la mortalidad fue muy baja (6.7\%) (Figura 1), sin embargo, se registraron niveles de 53, 67, 83, 90 y $96.7 \%$ de mortalidad para los tratamientos de $2.8,5.5,8.3,11$ y $13.8 \mathrm{mg} / \mathrm{L}$, respectivamente a las 96 horas de su aplicación. La baja mortalidad registrada en el grupo control (6.7\% a las 96 horas) permite inferir que las condiciones experimentales no tuvieron mayor incidencia sobre la mortalidad de los individuos tratados [16].

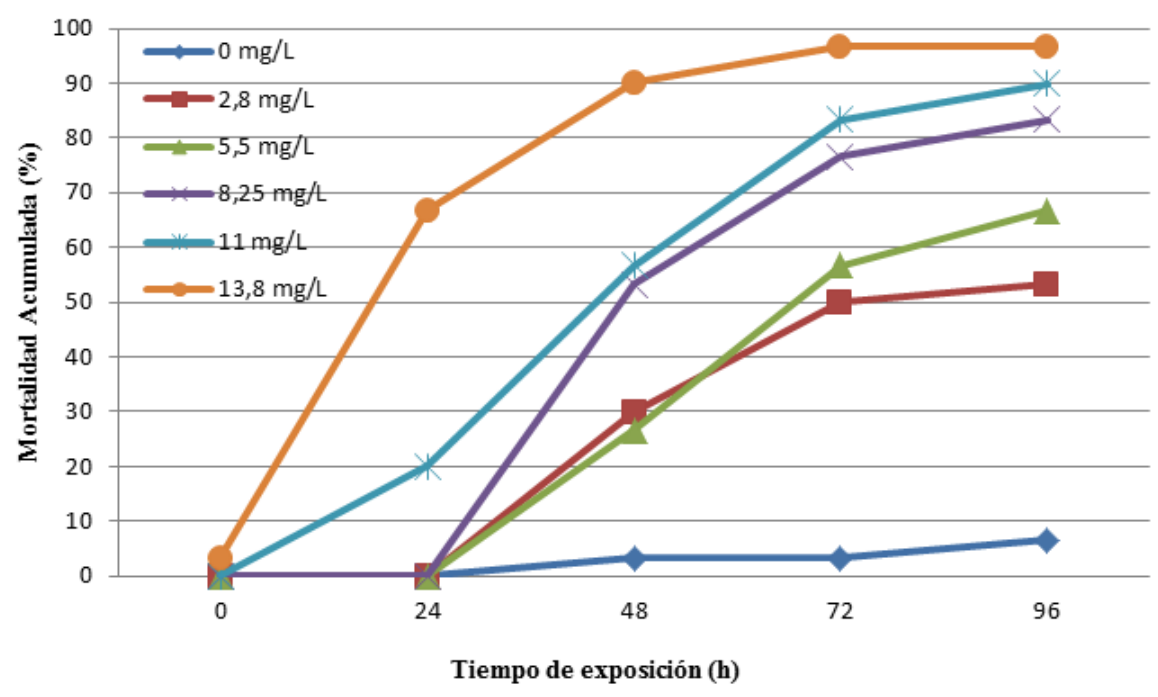

Figura 1. Mortalidad acumulada de los peces en el tiempo para las diferentes concentraciones de glifosato evaluadas.

Los dos tratamientos con mayor concentración de glifosato mostraron diferencias con respecto al testigo y a los tratamientos menos concentrados desde las primeras 24 horas. A las 48 horas todos los tratamientos con glifosato muestran un porcentaje de mortalidad alto con respecto al testigo. Al analizar los datos estadísticamente a las 96 horas (Figura 2) se observó una diferencia estadísticamente significativa entre cada uno de los tratamientos y el testigo con una confiabilidad del $95 \%$ (del $92 \%$ en el caso de la comparación entre el testigo y el tratamiento de $2.8 \mathrm{mg} / \mathrm{L}$ de glifosato). Los resultados estadísticos muestran tres grupos estadísticamente iguales entre sí y diferentes a los demás grupos (Figura 2): el primero es el testigo, el segundo los tratamientos de 2.8 y 5.5 $\mathrm{mg} / \mathrm{L}$ y el tercero los tratamientos de $8.25,11$ y $13.8 \mathrm{mg} / \mathrm{L}$. De este análisis se deduce que una concentración baja de glifosato $(2.8 \mathrm{mg} / \mathrm{lt})$ ya tiene un efecto significativo en la mortalidad de los individuos. A partir de la dosis $8.25 \mathrm{mg} / \mathrm{L}$ las mortalidades a las 96 horas son tan altas que ya no muestran diferencias estadís- 
ticamente significativas con respecto a los tratamientos con mayor concentración de glifosato.

\begin{tabular}{|c|c|c|c|c|c|}
\hline & $0 \mathrm{mg} / \mathrm{l}$ & $2.8 \mathrm{mg} / \mathrm{l}$ & $5.5 \mathrm{mg} / 1$ & $8.25 \mathrm{mg} / \mathrm{l}$ & $11 \mathrm{mg} / \mathrm{l}$ \\
\hline $2.8 \mathrm{mg} / 1$ & $\begin{array}{l}\mathrm{Si}{ }^{*} \\
\mathrm{P}=0.072\end{array}$ & & & & \\
\hline $5.5 \mathrm{mg} / 1$ & $\begin{array}{l}\mathrm{Si} \\
\mathrm{P}=0.013\end{array}$ & $\begin{array}{l}\text { No } \\
P=0.3653\end{array}$ & & & \\
\hline $8.25 \mathrm{mg} / \mathrm{l}$ & $\begin{array}{l}\mathrm{Si} \\
\mathrm{P}<0.000\end{array}$ & $\begin{array}{l}\mathrm{Si} \\
\mathrm{P}=0.07\end{array}$ & $\begin{array}{l}\mathrm{Si} \\
\mathrm{P}=0.039\end{array}$ & & \\
\hline $11 \mathrm{mg} / \mathrm{l}$ & $\begin{array}{l}\mathrm{Si} \\
\mathrm{P}<0.000\end{array}$ & $\begin{array}{l}\mathrm{Si} \\
\mathrm{P}=0.01\end{array}$ & $\begin{array}{l}\mathrm{Sí} \\
\mathrm{P}=0.008\end{array}$ & $\begin{array}{l}\text { No } \\
P=0.409\end{array}$ & \\
\hline $13.8 \mathrm{mg} / \mathrm{l}$ & $\begin{array}{l}\mathrm{Si} \\
\mathrm{P}<0.000\end{array}$ & $\begin{array}{l}\mathrm{Si} \\
\mathrm{P}<0.000\end{array}$ & $\begin{array}{l}\mathrm{Si} \\
\mathrm{P}=0.002\end{array}$ & $\begin{array}{l}\text { No } \\
P=0.112\end{array}$ & $\begin{array}{l}\text { No } \\
P=0.409\end{array}$ \\
\hline
\end{tabular}

Figura 2. Resultados de la comparación de medias de la mortalidad de P.reticulata sometida a cuatro concentraciones de glifosato comercial,a las 96 horas, mediante la prueba Kruskal Wallis. ${ }^{\star}$ Sí existe diferencia con una confiabilidad del $90 \%$. Tomando una confiabilidad del $95 \%$ el testigo y el tratamiento de $2.8 \mathrm{mg} / 1$ son estadísticamente iguales.

Al ajustar los datos a un modelo logístico se encontró un valor de $3.14 \mathrm{mg} / \mathrm{L}$ para la $\mathrm{CL}_{50-96}$, este es un valor mucho menor que el que se reporta en la literatura para otras especies [2] Efectivamente la concentración letal 50 para alevinos de cachama blanca (Piaractus brachypomus) es de $97.47 \mathrm{mg} / \mathrm{L}$ [11], para truchas de diferentes especies (Oncorhynchus spp) entre 10 y $190 \mathrm{mg} / \mathrm{L}$ [20], para perca (Leptomis macrochirus) $120 \mathrm{mg} / \mathrm{L}$ [9]. Este valor tan bajo puede ser atribuido en parte a que no se está evaluando el glifosato puro sino que el glifosato en la formulación del Roundup® 747 SG, que además del ingrediente activo contiene surfactantes y excipientes que también pueden ser tóxicos en peces [9], este estudio no permite por tanto determinar cuál es la contribución específica del ingrediente activo y cuál es la de los otros elementos contenidos en la formulación comercial. P. reticulata podría entonces tener un potencial interesante para desarrollar protocolos de evaluación en campo que permitan confirmar la contaminación de cuerpos de agua cuando existan actividades que hagan uso de glifosato, como aplicaciones aéreas en cultivos lícitos como ilícitos. Sumado a las investigaciones realizadas por otros autores [2], [11], [9], [20] el presente trabajo evidencia la sensibilidad de los peces al glifosato, principalmente en individuos jóvenes, lo que en el ambiente natural puede conducir a desbalances ecológicos en los ecosistemas. 


\section{Conclusiones}

Poecilla reticulata es una especie que evidencia una alta sensibilidad a la exposición aguda a glifosato en su presentación comercial Roundup® 747 SG. Así, concentraciones de $2.8 \mathrm{mg} / \mathrm{L}$ son suficientes para causar mortalidades altas experimentalmente y esta especie registró una $\mathrm{CL}_{50-96}$ de $3.14 \mathrm{mg} / \mathrm{L}$. Esta susceptibilidad de la especie sumada a otras ventajas de manejo y obtención de especímenes y la posibilidad de ser evaluada para otros xenobioticos.

La baja $\mathrm{CL}_{50-96}$ de $3.14 \mathrm{mg} / \mathrm{L}$, encontrada en este experimento refuerza la noción que el glifosato, puede tener un impacto negativo sobre los ecosistemas acuáticos, siendo así que dosis muy bajas pueden causar mortalidad de las especies de peces pequeños lo que impacta las cadenas tróficas de los mismos. Para desarrollar protocolos de bioindicación de contaminación por glifosato usando especímenes de $P$. reticulata es necesario determinar la sensibilidad de esta especie tanto al glifosato puro como a los excipientes y coadyuvantes más comunes en las formulaciones comerciales.

\section{Referencias}

1. Murtaza Alibhai and William Stallings, Closing down on glyphosate inhibition with a new structure for drug discovery, Proceedings of the National Academy of Sciences of the United States of America, vol. 98, no. 6, pp. 2944-2946, March 1991.

2. WHO, International Programme on Chemical Safety. Glyphosate, Environmental health criteria 159, Geneve, 1994.

3. CONICET, Evaluación de la información científica vinculada al glifosato en su incidencia sobre la salud humana y el ambiente, Argentina, 2009.

4. Iang Rondón, Wilson Ramírez, and Pedro Eslava, Evaluación de los efectos tóxicos y concentración letal 50 del surfactante Cosmoflux $411 \mathrm{~F}$ sobre juveniles de cachama blanca (Piaractus brachypomus), Revista Colombiana de Ciencias Pecuarias, no. 20, pp. 431-446, 2007.

5. S.O Duke and S.B. Powles, Glyphosate: a once in a century herbicide, Pesticides Management Science, no. 64, pp. 319-325, 2008.

6. UNOC, Censo de cultivos de coca 2005 Colombia, monitoreo de cultivos de coca, Bogotá, 2006.

7. UNOC, Colombia: monitoreo de cultivos de coca 2009, Bogotá, 2010.

8. Pedro R. Eslava, Wilson F. Ramírez, and Iang S. Róndon, Sobre los efectos del glifosato y sus mezclas: Impacto en peces nativos, Orinoquia, Colombia, 2007.

9. L.C. Folmar, H.O. Sanders, and A.M. Julin, Toxicity of the herbicide glyphosate and several of its formulations to fish and aquatic invertebrates, Archives of Environmetal Contamination and Toxicology, no. 8, pp. 269-278, 1979.

10. M.L. Morgan and J.W. Kiceniuk, Response of rainbow trout to a two month exposure to Vision, a glyphosate herbicide., Bulletin of Environmental Contamination and Toxicology, vol. 48, pp. 772-780, 1992.

11. Wilson Ramírez-Duarte, Iang Rondón-Barragan, and Pedro Eslava-Mocha, Acute toxicity and histopathological alterations of Roundup herbicide on cachama blanca (Piaractus brachypomus), Pesq. Vet. Bras., vol. 28, no. 11, pp. 547-554, 2008 Noviembre. 
12. Iang Rondón-Barragan, Glyphosate (Roundup) and Cosmoflux 411F induce oxidative stress in red-bellied pacu (Piractus brachypomus), Orinoquía, vol. 16, pp. 162-176, 212.

13. Edwin Gomez-Ramírez, Leslie Guzmán-Beltran, Pedro Eslava-Mocha, and Hernán Hurtado-Giraldo, Efecto de dosis subletales de un herbicida comercial en el sistema nervioso de alevinos de cachama blanca (Piaractus branchypomus), Orinoquia, vol. 16, no. 2, Diciembre 2012.

14. Patricia Devezé, Juan Lorenzo Reta, and Basilio Sánchez, Cultivo de Poecilla reticulata (Pisces: Poecilidae) en cuerpos de agua tropicales, Veracruz, México, Revista de biología tropical, vol. 52, no. 4, pp. 951-958, 2004.

15. R. L. Welcomme, International introductions of inland aquatic species, FAO fisheries tecnical paper, no. 294, 1988.

16. OCDE (Organización para la Cooperación y el Desarrollo Económico), Fish acute toxicity test, in OECD guideline for testing of chemicals., 1992.

17. R.J. Menéndez-Helman, G.V. Ferreyroa, M. dos Santos Alfonso, and A. Salibián, Glyphosate as an acetylcholinesterase inhibitor in Cnesterodon decemmaculatus, Bulletin of Environmental Contamination and Toxicology, vol. 88, no. 1, pp. 6-9, 2012.

18. Nicole Roy, Bruno Carneiro, and Jeremy Ochs, Glyphosate induces neurotoxicity in zebrafish, Environmental Toxicology and Pharmacology, pp. 45-54, Enero 2016.

19. Samanta Palas, Pal Sandipan, Kumar Murkherjee Aloke, and Ratan Ghosh Apurba, Biochemical effects of glyphosate based herbicide, Excel Mera 71 on enzyme activities of acetylcholinesterase (AChE), lipid peroxidation (LPO), catalase (CAT), glutathione-S-transferase (GST) and protein content on teleostean fishes, Ecotoxicology and Environmental Safety, vol. 107, pp. 120-125, September 2014.

20. M T Wan, R G Watts, and D J Mould, Effects of difernt dilution water types on te acute todicity of juvenile pacific salmonids and rainbow trout of glifosate and its formulated products, Bulletin of Environmental Contamination and Toxicology, vol. 43, no. 3, pp. 378-385, 1989. 